\title{
Design Thinking using the Blockchain
}

\section{Enable Traceability of Intellectual Property in Problem-Solving Processes for Open Innovation}

\author{
Alexander Schönhals, Thomas Hepp, Bela Gipp \\ Department of Computer and Information Science \\ University of Konstanz \\ Konstanz, Germany \\ \{firstname.lastname\}@uni-konstanz.de
}

\begin{abstract}
In this vision paper, we present an approach that makes it possible to protect developed ideas and early concepts even during their systematical development. We take the Design Thinking process as an example, in which interfaces are used for individual stages (understand, observe, define, ideate, prototype, test) to digitally record verbal, written or sketched, and even modeled or constructed outcome. This outcome is recorded and linked to the originating person. To guarantee both proof-of-existence and proof-of-origin, a unique hash is generated from each digital artifact stored and embedded into the Bitcoin Blockchain by the OriginStamp decentralized trusted timestamping service. Once this unique fingerprint is embedded in a transaction in the underlying Blockchain network, it can be proven where particular contributions originated due to the characteristics of Blockchain architecture. By setting up a decentralized tamper-proof means of record keeping, the entire innovation chain from the first ideation to the beginning of production is verifiably stored. By providing a clear proof-of-origin, all innovators (even competitors) could continue to work on existing problem-solving process and add their contribution proportionately, depending on the state of innovation development. This concept enables an Open Innovation ecosystem, which has the potential to increase the innovation potential of companies immensely. Additionally, inventions that are not patentable because they do not comply with the strict regulations of patent law can still be published and protected because the information about the origin of the respective contribution is guaranteed.
\end{abstract}

\section{KEYWORDS}

blockchain applications, design thinking, intellectual property, open innovation

\section{INTRODUCTION}

Over the past decades, the ability to innovate and the speed with which companies embrace innovation have became a central aspect in the long-term competitiveness of companies. Today's quickly changing market forces companies to adapt more flexibly, which is made possible by promoting and adopting innovative solutions. A company which underestimates innovation cannot exist in the long term [8]; Hence, innovation ensures survival.

While in the past, innovations have been produced organically, nowadays to increase the innovation potential, more and more companies aspire an innovation culture. The aim is to solve prevailing problems in a more systematic and user-centric way. Customer needs must be recognized at an even earlier stage. The early involvement of customers in the innovation cycle thus plays an important role. It is a clear objective at the early stage of innovation cycle to facilitate interactive relationships between all participants in the innovation process [19]. The concept of Open Innovation defined in 2003 by Chesbrough et al. stands for this opening of the innovation process. But how can the process be opened, when the first ideas and early concepts must be kept secret within an organization to not jeopardize its competitiveness?

Innovations often go hand in hand with problem-solving, so that in the past a large number of problem-solving processes were presented $[2,5]$. One of the most popular problem-solving processes has become known almost parallel to the Open Innovation movement - the Design Thinking (DT) methodology. Even though it was actively researched in the 1980s and 1990s [6, 20], a public breakthrough occurred when Stanford University began teaching 'Engineering Design Thinking' in 2005 [11]. This approach, with a focus on business purposes, spread like wildfire through various academic institutions and large for-profit companies. Even if this model introduces a target-oriented structure into creative creation in the innovation process, it is still difficult to measure the true impact on innovation culture, as with all problem-solving processes [23]. Problem-solving methods are by definition iterative, and their results are not deterministic. These characteristics make it difficult to measure the added value by using systematical generation of ideas to increase innovation. As a result, the innovative 
strength of institutions and corporations is usually measured by the number of patents applied for and disclosed or the content of their innovation portfolio, which is never fully communicated to the public.

Patents only represent the end outcome and do not document the 'evolution' of a particular invention. Inventions rarely originate from one individual person, and in patent applications, the respective contribution of multiple inventors must be negotiated, which can often lead to disputes, since the respective individual inputs do not seem to be measurable in retrospect.

Patent protection has made a major contribution to the culture of innovation, especially in industrialized countries, but it should be noted that the long development process of a particular invention or patent is not subject to protection and therefore the whole process often takes place in a 'chamber of secrets'. Nevertheless, the development of ideas would benefit from external impulses even at an early stage. However, the development is kept secret, because they fear that the whole idea will be abused or stolen.

The remainder of the paper is structured as follows: First, we give an overview of the related work focusing protection of intellectual property on the Blockchain. Then, we introduce the approach of enabling traceability of recorded intellectual property assets. Next, we provide a more detail insight into our proposal using the example of design thinking method. We conclude the paper with some limitations, further research opportunities.

\section{RELATED WORK}

This section introduces the areas directly related to the approach of Design Thinking using the Blockchain. These include the methods for measuring the impact of problem-solving processes and the work that has been presented so far to increase innovation potential. Furthermore, we provide an overview of the approaches to intellectual property (IP) protection using Blockchain technology (BT).

\subsection{Measuring the Impact of DT}

Several promising approaches were developed to quantify an innovation process, and its outcome, e.g., the measurement of the total number of patents or patent applications through an ideation workshop was presented by Ylitalo [25]. This study reveals that the implementation of ideation sessions is extremely cost-effective. At an ideation workshop, the employees generate 40 times the number of invention and patent applications for the same duration compared to the general formulation of an invention into a web-based platform.

However, the study also revealed that not all employees report their ideas in the workshop itself but often after a particular workshop. One of the reasons for this behavior is that the development of ideas in the workshop is in a plenary session and therefore it is difficult to determine the exact origin of an idea. It is assumed that employees report ideas after a workshop to isolate their idea. Supporting evidence for this theory is that after a workshop, a significant increase has been observed in the number of invention reports into web-based platforms.

The introduced study considered all-day workshops that were in fact based on the DT model but focused only on the ideation stage.

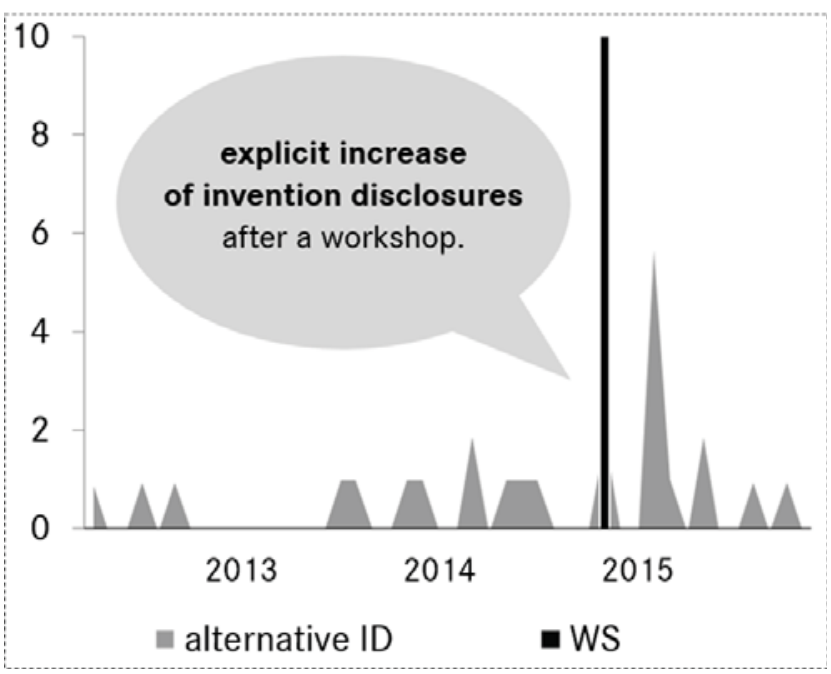

Figure 1: This diagram shows the impact of an innovation workshop (WS) to invention disclosures (ID) inside a company of an example-workshop-group, $n=35$. The Figure was adapted by Ylitalo [25]

Hence, essential elements such as the definition of the problem and prototyping were excluded in this process.

Even though the measurement is cumbersome, attempts have been described that measure the impact of DT, e.g., Schmiedgen et al. [23] from the Hasso Plattner Institute of Design Thinking. Approximately 400 participants from for-profit enterprises participated in the study. The group evaluated both qualitative feedback queries and integrated quantitative sales figures of innovations. Mainly execution-oriented metrics were pursued. Whereas the group from the d.school at Stanford University [21] pursued a creativity-oriented approach. Understandably, Royalty and Roth mention that the exclusive use of execution-based metrics would unsettle DT participants, as only behaviors that lead to better execution in the sense of being more efficient but not novel are rewarded.

Determining whether a problem-solving process influences innovation is described in both papers as cumbersome. The main causes are the lack of traceability $[18,23]$ of individual ideas, as well as the quite high portion of subjective decisions in the creative process.

\subsection{IP Protection using the Blockchain}

The Blockchain is a promising technology. Due to its integrity and immutability characteristics, BT affects almost all businesses where digital transactions are executed, and a high level of trust is required. In summary, its architecture is predestined for the protection of intellectual property. A joint study The Future of Intellectual Property by the Dennemeyer Group and the trend research institute 2bAHEAD [7] dealt in particular with the developments in recent years and how relevant authorities across the world, such as patent and trademark offices, are struggling to keep pace with the continued and increased volume of industrial property right requests in the digital age. The study offers 28 strategic recommendations for companies. Point 23, for example, states:"Together with partners, 
create a Blockchain-based alternative for conventional IPR that is quicker, more transparent, impossible to manipulate, and works without middleman".

A scientific discussion on this topic is provided by the work of de la Rosa et al.. The holistic survey analyzes existing and future applications, which deal in particular with IP management and Open Innovation $[9,10]$. Among other things, they are focused on the recently released approach of decentralized trusted timestamping (DTT) [14]. In this approach, a unique hash, e.g., SHA-256 is generated from a digital document and concatenated into a transaction embedded in a Blockchain together with a prior generated timestamp. Due to the properties of most Blockchains, e.g., Bitcoin, Ethereum, etc., it is computationally hard to manipulate this embedded transaction code afterward. Based on this kind of notarization service, all IP related functionalities can make use of the BT, e.g., record keeping, IP protection [3, 4], licensing by smart contracts, NDA management, access control, traceability and rewarding.

\section{ENABLE TRACEABILITY OF IP}

A body of thought can only be protected if it is first expressed and secondly recorded on a medium. In the past, ideas and inventions were recorded on paper and affixed with a seal by an intellectual property office (IPO). The digitalization of early concepts and ideas is not new. There has been done a lot of work for decades to offer intuitive and user-friendly solutions. Nevertheless, ideas developed from employees are nowadays mostly entered via web forms into internal idea collection platforms (intranet). Often after an innovation workshop using creativity techniques, but not during a creative work.

In the following sections, we discuss the features of capturing ideas during a DT session. After we explain how the uttered content is currently being digitized. The aggregation process and the preserving process are explained to illustrate how we realize our idea protection process.

\subsection{Recognition and Capturing}

Capturing idea history paths is not a new idea, but this was done typically in a textual way, using web-based platforms mainly for reuse purposes [13]. In this subsection, we do not want to concentrate on conventional web-based capture, but rather look at technologies and approaches that digitize the outcome at most with a slight distraction of the innovator during or immediately after a certain phase of the DT process. The attention at the recording process is set to not affect the usual behavior of participants. During DT workshops the utterance of ideas and thoughts takes place on different channels. In unstructured observations of conducted DT workshops with the aim of generating ideas, five channels were identified, i.e. verbal, non-verbal, written or sketched, modeled or constructed, performed. Each of these outcome channels can nowadays be digitized using state-of-the-art technologies.

Verbal. Much research has been performed in the field of spoken term detection (STD) [17, 22].

Discerning several voices in a room can also be achieved by pattern recognition [16]. In the context of innovation generation, criteria must be identified to extract specific insights, e.g., the measurement of speech content in a discussion to determine the share of a contribution in a particular session.

Non-verbal. The non-verbal channel is detected by conventional RGB cameras, IR cameras or ultrasonic sensors. There are many ways in which humans communicate using gestures and facial expressions, but also by moving the body [24, 26]. There is a common state of knowledge that gestures complement not only semantic information to the verbal utterance, but that gestures can completely invert a spoken word. Therefore, this awareness is regarding the formulation of ideas and concepts of special interest [12]

Written or sketched. During the DT session keywords are written on analog paper and drawings or sketches are made. These scripts can be digitized using ordinary scanning. By post-processing using optical character recognition (OCR) and transformation in scalable vector graphics, the data can be aggregated uniformly. Digital pens are already being used during a creative session so that every note in the process of creation is digitized directly. This input also enables us to recommend or suggest existing solutions or innovation processes, which are taken place at the same time even in real-time.

Modeled or constructed. A special focus in the DT process is the handling of three-dimensional objects. Prototypes are produced in different levels of detail. Digitizing prototypes is a major challenge. With 3D-scanning objects from the real-world can be reconstructed computer-aided as CAD-models. Since each prototype can consist of a wide variety of materials, depending on the problem definition and solution approach, the digital 3D-object can only be reconstructed to a limited extent afterward. Last, digitizing prototypes is classified as one of the biggest challenges in capturing outcome during a DT process.

Performed. In certain cases, ideas cannot be communicated verbally or in written form. In this case, an idea is re-enacted or staged. Digitizing a performance differs from non-verbal only in the intention of the participants of a session. They disguise themselves and thus play a role. This has to be taken into account when recording. As we see, a few channels can capture participants' comments in real time, while other channels are not suitable for digitization until later. We suspect that the more channels are tapped, the more precisely the author of a contribution can be identified by an identity recognition system. The next section explains how the data is aggregated and how the OriginStamp service can be used to make idea origination traceable.

\subsection{Protection of IP with OriginStamp}

Gipp et al. presented an approach to timestamp any digital content [14]. With the help of this approach, the hash of any captured and digitized DT outcome, which is enhanced by meta information, e.g., originator, session, geo-location, etc. is embedded into the underlying Blockchain.

During the ideation process, many different types of digital content are created. This includes pictures, sketches, voice memos, documents or mind maps. Since idea generation is an iterative process, these documents must be made traceable to understand the individual contributions of the users. To ensure the integrity of 


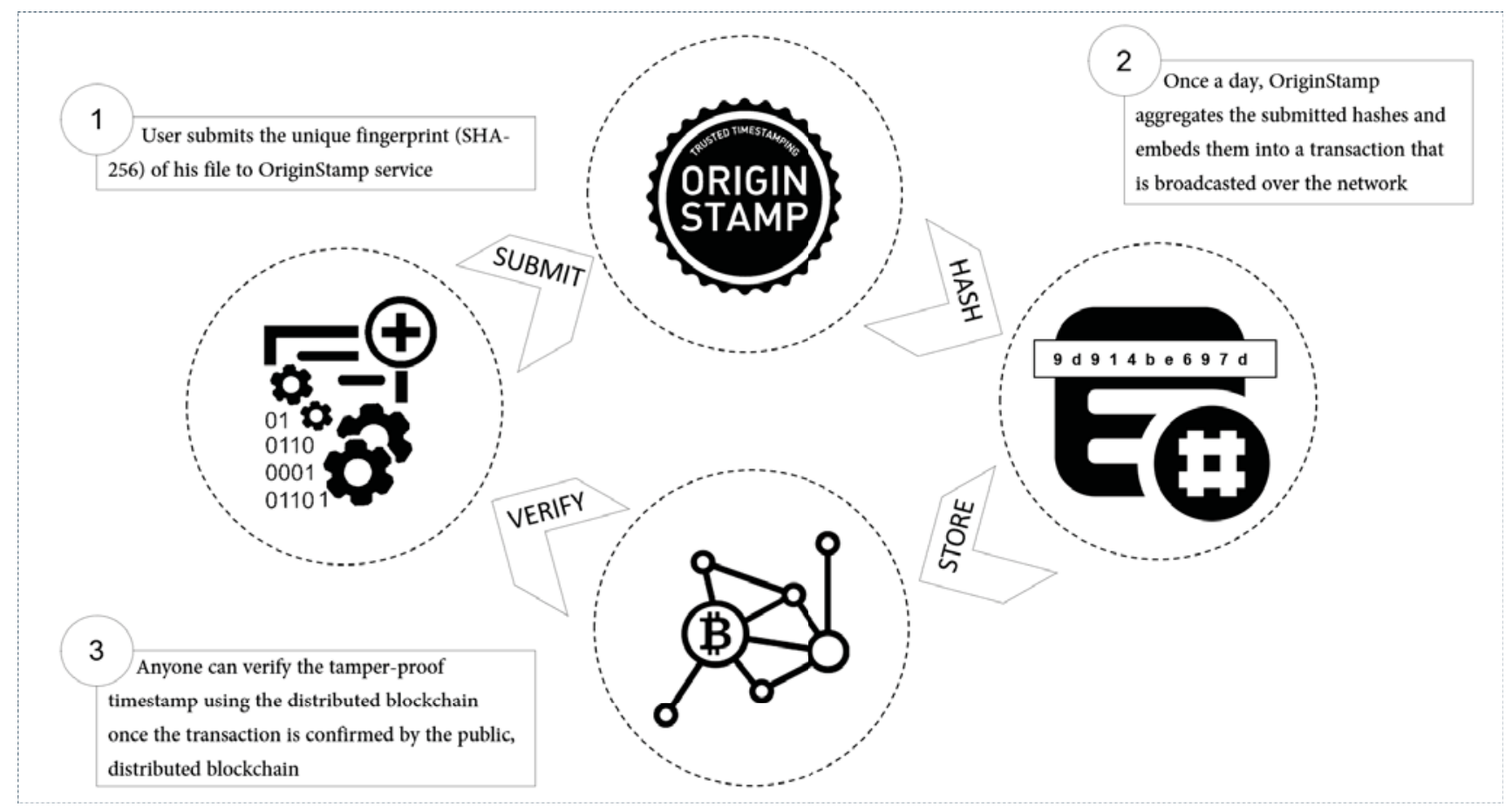

Figure 2: This figure illustrates a brief overview of the OriginStamp service. (http://www.originstamp.org)

the data later on, e.g., to show that the contributions are complete and have not been manipulated, we use decentralized trusted timestamping, which was introduced by Gipp et al. in 2015 [14]. Figure 2 shows the trusted timestamping workflow, which begins with the determination of a unique fingerprint, a so-called hash. A hash function calculates a unique fingerprint that has a fixed size, of digital content, regardless of its size. The most important characteristic is that it is impossible to decode the hash to its original content. This means that ideas, in any form, can be protected at an early stage without publishing them.

In our concept, we determine the SHA-256 [15] of the individual parts of an idea. These hashes are bundled and sent to OriginStamp via the RESTful interface, which is easy and free to use. OriginStamp could instantly submit the hash to the Bitcoin network through a transaction. However, since this is very cost inefficient and the Bitcoin Blockchain could only process seven transactions per second, all hashes are collected within a certain period. This period is currently set to 24 hours. As shown in Figure 2 the seed of these hashes is calculated once per period. To do this, the new hashes are sorted alphabetically and appended, which is the so-called seed. The seed is again hashed and used as a private key to generate a Bitcoin address. By broadcasting a transaction to this address, the timestamp is generated, as soon as this transaction is confirmed and mined into a block by the network. From this point in time, the existence of an idea or part of an idea can be verified by the original file and the seed independently from a central instance like [1].

The captured file of DT outcome conditioned with meta information and stored in a central database regarding the transaction.
Any progress in the innovation process is, as long as it can be digitized, also protected. The central database, where the captured objects are stored, is used in the Open Innovation context either by a consortium or public.

\section{DESIGN THINKING USING BLOCKCHAIN}

During the DT work many types of outcome are created, which are explained in detail in the Section 3.1. To adequately access the existing channels, interfaces must be identified and defined in the respective phases of the DT process. In addition to identifying interfaces for digitizing outcome and describing the individual steps to enable traceability, this chapter uses a big picture (Figure 3) to explain the work flow in detail using a single phase of DT model as an example and describes the essential steps of the tracking cycle.

\subsection{Interfaces in DT Stages}

Every phase of the DT process requires special interfaces that need to be evaluated. While in the very first phase, the understanding, one mainly accesses sources of inspiration and a knowledge base and generates little or no outcome, in the ideation phase a multitude of creative output is generated, and implications are gained. While one would benefit from real-time capturing in individual phases, such as the ideation phase, because recommendations or suggestions can be made directly and selectively on specific topics, real-time support during the prototyping phase is difficult to implement due to the variety of actions involved. 


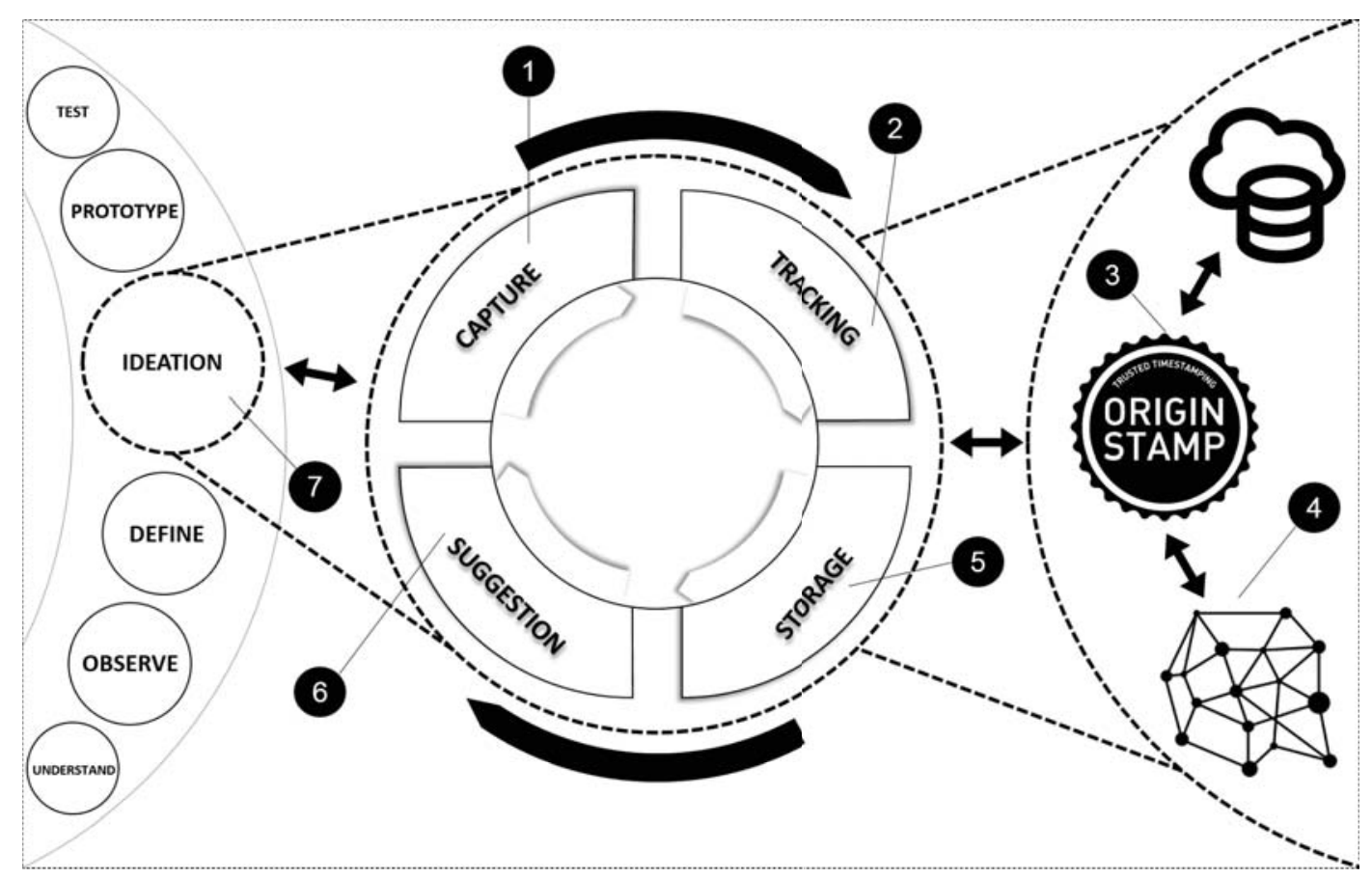

Figure 3: This Figure illustrates the work flow of IP record keeping during a design thinking process (ideation). (1) the outcome of ideation session is captured by technologies, e.g., digital pen. (2) digital innovation object (DIO) is created and supplemented by meta information, e.g., inventor, geo-location, prior version (3) fingerprint of DIO is generated and sent to OriginStamp via REST post (4) fingerprint is now embedded in the Blockchain - IP is protected now (5) DIO, as well as the fingerprint, is stored in the database (6) based on semantic input a related topic is determined (7) participants of DT session receives a suggestion or recommendation.

Nevertheless, investigations have to be carried out that reveal interfaces for any outcome. Then DT outcome has to be digitized to build up an exact course of innovation design progress.

\subsection{Dockable Tracking Cycle}

To digitally record output and outcome during the DT processes, we define a dockable tracking cycle (DTC) that can be docked to individual phases of the DT process. It iterates through sequential steps and processes not only outcomes generated by participants in the innovation process but also the insights gained from the information system itself. The DTC is divided into four steps, i.e. capturing, tracking, storage, and suggestion. These steps are explained briefly in the following subsections.

4.2.1 Capturing. In this phase, the behavior and utterance of participants are digitally recorded. These can be video or audio recordings which are post-processed with recognition algorithms to extract insights. In addition, recorded digital artifacts, which are used as the basis for further tracking are created in this phase.

4.2.2 Tracking. In this phase, recorded artifacts are supplemented by meta information and aggregated into a so-called digital innovation object (DIO). By using the identity management system, explicit contributors are identified for each recording and also stored in the DIO. We call the extension of recorded artifacts by linking existing and previous patents or ideas as conditioning. In the course of conditionings, duplicates are recognized and identified early on by text analysis and similarity algorithms and are also included in the DIO.

4.2.3 Storage. This phase is dedicated to the storage and protection of digital innovation objects. In this step, a unique hash of DIO is created. The fingerprint is then embedded on the Blockchain using the OriginStamp service. The DIO object is stored centrally in the database and can be accessed by all ecosystem participants. Proof-of-existence of a digital innovation object can be verified after the fingerprint is embedded on the Blockchain. The exact procedure is explained in Section 3.2.

4.2.4 Suggestion. Based on the outcome of the DT process, which includes keywords, unusual constellations within the group or any other insights gathered, recommendations or helpful hints are generated during this phase and passed on to the participants of the DT process. This is intended to boost the development of innovations at an early stage and possibly bring together already existing innovation developments in related subject areas.

\section{LIMITATIONS AND FUTURE WORK}

This concept offers many opportunities for further promotion of Open Innovation, as well as the protection of IP, but there are some limitations that should not go unmentioned. First, digital objects 
cannot be stored on the Blockchain due to the scalability issues. However, there is a lot of related work which discussed this issue.

Further limitations are non-technical and relate to patent law. An invention disclosure is subject to the first-to-file (FTF) system, the right to the grant of a patent for a given invention lies with the first person to file a patent application for protection of that invention, regardless of the date of actual invention. Hence, the trusted timestamp date is not relevant for a potential disclosure of an invention (at this juncture). Amendments to the law would be a prerequisite for replacing standard patents. As future work, the following steps are indispensable. It must be determined which technologies are suitable for the digital capture of idea formulations. This means that the inventor should not be distracted in any way during creative work by detection systems. A literature survey for technical methods for idea capturing will be created. The next step is to identify where interfaces are available for recording. Where are potential interfaces in Design Thinking process to capture and digitize outcomes, e.g., drawings, transcriptions, prototypes, test results, etc.

Further, an evaluation of decentralized storage solutions in respect of their scalability and security has to be done. To separate from the identity management of an enterprise, decentralized identity management must be considered which can also be realized with Blockchain technology. It must also be investigated how the Blockchain can be used as a mediator for the exchange of ideas across organizations.

\section{CONCLUSION}

The primary value of our approach is the protection of intellectual property which is directly linked to the identity of the originator even during the innovation development in real-time. The digitized version of the idea, the digital innovation object, can be indexed by a suggestion or recommendation system and more easily processed for the corresponding recommendations. Text analysis and similarity algorithms can be used to determine the tamper-proof point in time when the idea popped up within a particular stage of a DT session, e.g., ideation phase. Even if an idea was not considered in more detail within a session and therefore had no added value at this point, this idea could still be stored permanently. The longtime preserved ideas enrich the corporate memory and also enable the automated idea-recycling through the recommender system. Versioning would be achieved by an updating mechanism. Further, Blockchain technology enables a well thought-out reward system, which is mapped on so-called smart contracts. This would increase the motivation of participants due to incentives. The potentially resulting patents can be based on these traceable digital innovation objects because the originator would be verifiable known. In the end, employees would have an intrinsic motivation to share their ideas already during the innovation development process. The innovation potential of particular sectors could be boosted by opening innovation portfolios to a larger audience and potentially even to the public, thus promoting and realizing the vision of Open Innovation. Our concept creates the foundation for measuring the impact of problem-solving models by building a traceable recording of each progress state at each stage of the Design Thinking process. Furthermore, it makes sense to integrate the logged history of a particular innovation into the resulting product itself so that tracking of intellectual property becomes the initial point for tracing supply chain stations. For this purpose, one has to think about how digital fingerprints (hashes) could be integrated into a product or its material to treat each product as unique. In the end, to guarantee forgery protection.

\section{REFERENCES}

[1] C Adams. 2001. RFC 3161: Internet X.509 Public Key Infrastructure Time-Stamp Protocol (TSP). (2001), 1-26.

[2] Genrich Altshuller. 1996. And suddenly the inventor appeared: TRIZ, the theory of inventive problem solving. Technical Innovation Center, Inc.

[3] Corinna Breitinger. 2016. Using the Blockchain of Cryptocurrencies to Encourage Open Discussion and Sharing of Ideas. Master's thesis. Linnaeus University, Department of Media Technology.

[4] Corinna Breitinger and Bela Gipp. 2017. VirtualPatent-Enabling the Traceability of Ideas Shared Online using Decentralized Trusted Timestamping. In Proceedings of the 15th Int. Symposium of Information Science (2017).

[5] Forrest W Breyfogle III. 2003. Implementing six sigma: smarter solutions using statistical methods. John Wiley \& Sons.

[6] Richard Buchanan. 1992. Wicked problems in design thinking. Design issues 8, 2 (1992), 5-21.

[7] Michael Carl and Maria Luebcke. 2017. The Future of Intellectual Property. (2017).

[8] Henry Chesbrough et al. 2003. Open innovation. (2003).

[9] Josep Lluis de la Rosa, Denisa Gibovic, V Torres, Lutz Maicher, Francesc Miralles, Andrés El-Fakdi, and Andrea Bikfalvi. 2016. On Intellectual Property in Online Open Innovation for SME by means of Blockchain and Smart Contracts. In $3 r d$ Annual World Open Innovation Conf. WOIC.

[10] Josep Lluis de la Rosa, Victor Torres-Padrosa, Andrés el Fakdi, Denisa Gibovic, O Hornyák, Lutz Maicher, and Francesc Miralles. 2017. A Survey of Blockchain Technologies for Open Innovation. In 4rd Annual World Open Innovation Conf. WOIC. 14-15.

[11] Clive L Dym, Alice M Agogino, Ozgur Eris, Daniel D Frey, and Larry J Leifer. 2005. Engineering design thinking, teaching, and learning. fournal of Engineering Education 94, 1 (2005), 103-120.

[12] Farina Freigang and Stefan Kopp. 2016. How gestures tune meaning of multimodal utterances: Analyses of modifying functions. (2016).

[13] Renate Fruchter and Peter Demian. 2002. Knowledge management for reuse. In Proceedings of CIB w78 Conference, Aarhus School of Architecture, Denmark. 12-14.

[14] Bela Gipp, Norman Meuschke, and André Gernandt. 2015. Decentralized trusted timestamping using the crypto currency bitcoin. arXiv preprint arXiv:1502.04015 (2015).

[15] IETF. 2011. RFC 6234 - US Secure Hash Algorithms b(SHA and SHA-based HMAC and HKDF). (2011). https://tools.ietf.org/html/rfc6234

[16] Chul Min Lee and Shrikanth S Narayanan. 2005. Toward detecting emotions in spoken dialogs. IEEE transactions on speech and audio processing 13, 2 (2005), 293-303.

[17] Jonathan Mamou, Bhuvana Ramabhadran, and Olivier Siohan. 2007. Vocabulary independent spoken term detection. In Proceedings of the 30th annual international ACM SIGIR conference on Research and development in information retrieval. ACM, 615-622.

[18] Katie Rapp and Caitlin Stroup. 2016. How Can Organizations Adopt and Measure Design Thinking Process? (2016).

[19] Ralf Reichwald and Frank Piller. 2005. Open Innovation: Kunden als Partner im Innovationsprozess. Online verfügbar unter http://www. impulse. de/downloads/open innovation. pdf, zuletzt geprüft am 5 (2005), 2007.

[20] Peter G Rowe. 1991. Design thinking. MIT press.

[21] Adam Royalty and Bernard Roth. 2016. Developing design thinking metrics as a driver of creative innovation. In Design thinking research. Springer, 171-183.

[22] Murat Saraclar and Richard Sproat. 2004. Lattice-based search for spoken utterance retrieval. In Proceedings of the Human Language Technology Conference of the North American Chapter of the Association for Computational Linguistics: HLT-NAACL 2004.

[23] Jan Schmiedgen, Lea Spille, Eva Köppen, Holger Rhinow, and Christoph Meinel. 2016. Measuring the impact of design thinking. In Design Thinking Research. Springer, $157-170$.

[24] Leonid Sigal, Alexandru O Balan, and Michael J Black. 2010. Humaneva: Synchronized video and motion capture dataset and baseline algorithm for evaluation of articulated human motion. International journal of computer vision 87, 1-2 (2010).

[25] Pekka Ylitalo. 2016. Effectiveness metrics for idea generation. (2016).

[26] Zhengyou Zhang. 2012. Microsoft kinect sensor and its effect. IEEE multimedia $19,2(2012), 4-10$ 